\title{
Spectral Changes of Pleione - Start of a New Cycle?
}

\author{
L. Iliev \\ Institute of Astronomy and \\ Bulgarian National Astronomical Observatory "Rozhen", \\ 72 Tsarigradsko Shossee Blvd., \\ BG-1784, Sofia, \\ Bulgaria
}

\begin{abstract}
This is a preliminary report on spectral variability of Pleione during the present $\mathrm{Be}$ phase. The spectral activity of the star confirms the observed photometrical trend.
\end{abstract}

\section{Introduction}

Pleione is a well known Be-shell star with quasi-regular spectral changes. Its last transition between a well developed $\mathrm{Be} / \mathrm{shell}$ phase and a phase with strong hydrogen emission was reported by Iliev et al. (1988) and many other authors. Using a long set of photometrical observations of the star Sharov and Lyutyi (1997) have predicted a change of status of Pleione earlier than observed. Our recent spectral observations reveal the beginning of a new phase of spectral activity of the star.

\section{Observations}

The observations were carried out at the coude spectrograph of the $2 \mathrm{~m} \mathrm{RCC}$ telescope of the Bulgarian National Astronomical Observatory "Rozhen". The reciprocal dispersion used was approximately 0.1 A per pixel for all observations. For the observations until January 1997 the ISTA CCD camera with Electron chip was used. The observations from November 1998 and February 1999 were carried out with the more sensitive Photometric CCD camera.

\section{Results and discussion}

From the observations, which cover the period from 1992 to 1999 , we can investigate the behaviour of $\mathrm{H} \alpha, \mathrm{H} \beta$ and Fe II 5317 spectral lines, which were selected as sensitive indicators of the changes expected to take place in the circumstellar envelope of Pleione. After a slight increase from November 1992 until January 1993 (Fig. 1) the intensity of the $\mathrm{H} \alpha$ emission stayed relatively constant until the end of 1998 .

The peak intensity varied only by $2 \%$ during this period, showing a slight tendency of a decrease. The equivalent width above the continuum showed 


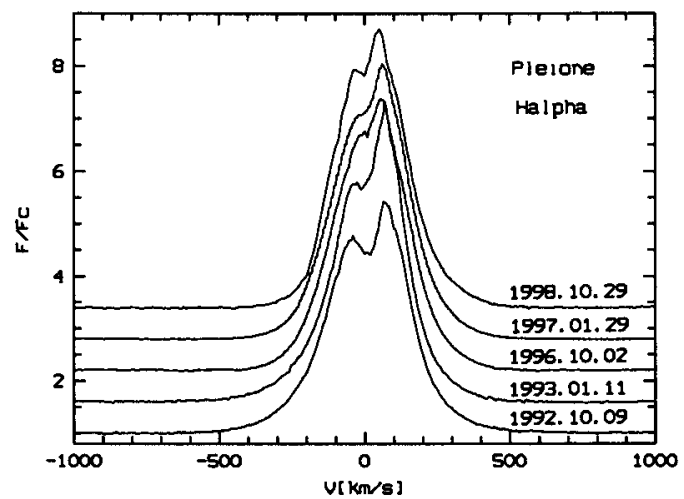

Figure 1. H $\alpha$ profile variations of Pleione

the same behaviour. In the October 1998 observations one can note a slight increase of the central absorption, resembling the one observed at the beginning of the Be-phase. The highest resolution observations of the recent observations (about 30000) reveal that the hydrogen emission profiles show a classical type 2 structure according to the scheme of Hanuschick et al. (1995) and fit well the model profiles presented for $\gamma$ Cas and $59 \mathrm{Cyg}$ (Hummel, 1998). It is interesting to note that this structure is present in $\mathrm{H} \beta$ as well (Fig.2).

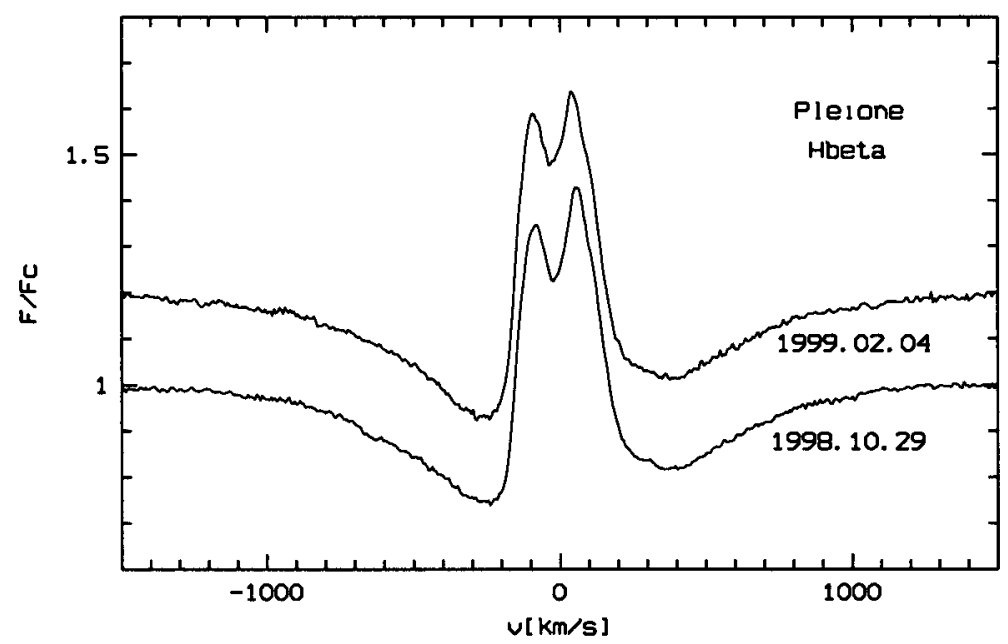

Figure 2. $\mathrm{H} \beta$ profiles

Compared with the profiles of Fe II 5317 from October 1996 - January 1997 (Iliev and Hoffman, 1997) (Fig. 3) we should note that V/R changes preceded the changes in the optically thick regions of the envelope of Pleione responsible for the emission in the Balmer lines. 


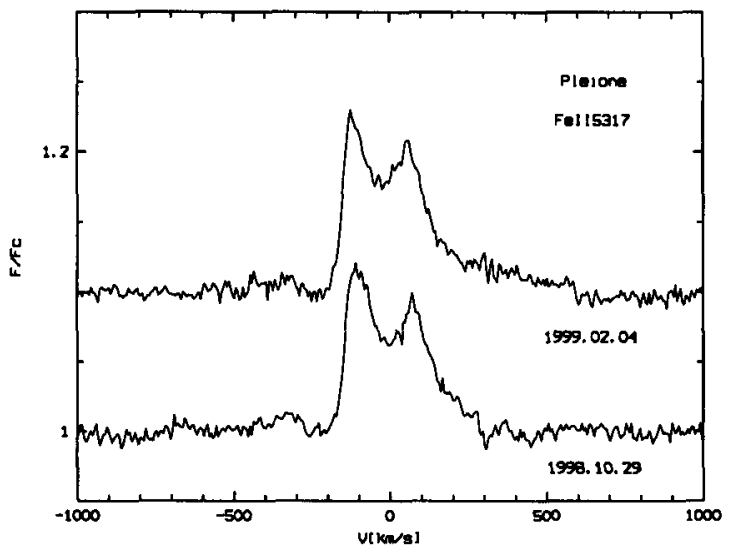

Figure 3. Fe II 5317 emission profiles

It has been shown that phase changes of Pleione are a consequence of geometry, size and temperature changes of the shell of the star (Zorec, 1994). Recent spectral activity of this always fascinating Be-star could well be used to explore the inner structure of the circumstellar envelopes of Be stars.

Table 1. Observations and spectral data

\begin{tabular}{ccccccc}
\hline Date & HJD $(2400000+)$ & Sp. region & V/R & Ie/C & FWHM & W $\lambda / \AA$ \\
\hline 1992.10 .09 & 48904.5952 & H $\alpha$ & 0.820 & 5.43 & 6.55 & 31.9515 \\
1993.01 .11 & 48999.2748 & H $\alpha$ & 0.754 & 6.71 & 5.65 & 34.5718 \\
1996.10 .02 & 50359.4364 & H $\alpha$ & 0.873 & 6.44 & 6.2 & 34.1850 \\
1997.01 .29 & 50478.4352 & H $\alpha$ & 0.813 & 6.23 & 5.98 & 34.1597 \\
1998.10 .29 & 51116.4309 & H $\beta$ & 0.821 & 1.42 & & \\
1998.10 .29 & 51116.5004 & Fe II 5317 & 0.715 & 1.118 & & \\
1998.11 .02 & 51119.6242 & H $\alpha$ & 0.863 & 6.24 & 5.88 & 33.5523 \\
1999.02 .04 & 51213.4163 & H $\beta$ & 0.883 & 1.430 & & \\
1999.02 .04 & 51213.4408 & Fe II 5317 & 0.799 & 1.129 & & \\
\hline
\end{tabular}

\section{References}

Hanuschik, R.W., Hummel, W., Dietle, O., Sutorius, E. 1995, A\&A 300, 163

Hanuschik, R.W., Hummel, W., Sutorius, E., Thimm, G. 1996, A\&AS 116, 309 Hummel, W. 1998, A\&A 330, 243

Iliev, L., Ruusalepp, M., Kovachev, B. 1988, Commis. 27 IAU Inf. Bull. Var. Stars, No 3204

Iliev, L., Hoffmann, D., 1997, unpubl.

Sharov, A.S., Lyutyi, B.M. 1997, Pis'ma Astr. Zh. 23, No 2, 113

Zorec, J. 1994, in IAU Symp. 162, L.A. Balona, H.F. Henrichs, J.M. Le Contel, Eds., Kluwer, p. 362 